\title{
Algorithm for Enhancing the QoS of Video Traffic over Wireless Mesh Networks
}

\author{
Abdul Nasser A. Moh ${ }^{1}$, Borhanuddin Bin Moh. Ali ${ }^{4}$ \\ Department of Computer and Communication Systems \\ Engineering, Faculty of Engineering \\ University Putra Malaysia Serdang 43400, Selangor, \\ Malaysia \\ Radhwan Mohamed Abdullah ${ }^{2}$ \\ Division of Basic Sciences, College of Agriculture and \\ Forestry, University of Mosul, Mosul, Iraq
}

\author{
Abedallah Zaid Abualkishik ${ }^{3}$ \\ American University in the Emirates \\ Dubai, United Arab Emirates
}

\author{
Ali A. Alwan ${ }^{5}$ \\ Department of Computer Science \\ Kulliyyah of Information and Communication Technology \\ International Islamic University Malaysia \\ Kuala Lumpur 53100, Malaysia
}

\begin{abstract}
One of the major issues in a wireless mesh networks (WMNs) which needs to be solved is the lack of a viable protocol for medium access control (MAC). In fact, the main concern is to expand the application of limited wireless resources while simultaneously retaining the quality of service $(\mathrm{QoS})$ of all types of traffic. In particular, the video service for real-time variable bit rate (rt-VBR). As such, this study attempts to enhance QoS with regard to packet loss, average delay, and throughput by controlling the transmitted video packets. The packet loss and average delay of QoS for video traffic can be controlled. Results of simulation show that Optimum Dynamic Reservation-Time Division Multiplexing Access (ODR-TDMA) has achieved excellent utilization of resource that improvised the QoS meant for video packets. This study has also proven the adequacy of the proposed algorithm to minimize packet delay and packet loss, in addition to enhancing throughput in comparison to those reported in previous studies.
\end{abstract}

Keywords-Wireless Mesh Networks (WMNs); Medium Access Control (MAC); Quality of Service (QoS); video traffic

\section{INTRODUCTION}

The importance of medium access control (MAC) protocol for wireless mesh networks (WMNs) is dependent on several reasons, such as enabling statistical multiplexing for wireless access interface, optimizing the use of limited wireless resources, and ensuring that the required quality of service (QoS) for multimedia packets are fulfilled, particularly for video service with real-time variable bit rate (rt-VBR) [1]. The assignment of dynamic slots is important to ensure that the achievement of statistical multiplexing in various service categories and features are adequate for variable bit rate (VBR) class that coordinates the vast traffic needs for both spatiallydispersed and independent wireless terminals [2-4]. There are several weaknesses in the present protocols for MAC; for instance residual lifetime, excessive overhead for transmission of buffer data, instant queue length, and punctual arrival of packet as an essential threshold. Two types of access schemes can be employed for transmission of data from individuallydistributed wireless terminals to contention less and contention-based access points. In the effort to diminish packet loss as a result of collision, the contention-based system [5-8] requires smaller control packet and lower collision probability. As such, several MAC protocols were developed for application in wireless networks. For instance, a comparative analysis has been carried out which involved two MAC protocols, namely RI_MAC (a contention-based receiver that initiates the asynchronous duty cycle in the MAC protocol) and ATMA (advertisement-based Time Division Multiplexing Access (TDMA) MAC protocol) [9]. A system that is free from any contention [10 -13] was described as being based on either a polling mechanism that assigns an uplink in the slot to transfer the parameters or a piggyback approach where the parameters are piggybacked on uplink data burst. The polling period must be altered to reduce loss of packet while the overhead of the piggyback has to be reduced. An MAC protocol based on TDMA was developed to prevent collision during data transfer in order to maintain QoS in networks with ad-hoc feature [14]. Other researchers have developed procedures for adaptive registration of mobile-to-mobile (M2M) networks with massive Internet of Things (IoT) devices. This was followed by the development of an MAC protocol with hybrid-slotted Carrier Sense Multiple Access/TDMA (CSMA/TDMA) (HSCT), in which the logical frame comprises two parts: the first part is a contention-based CSMA with a collision avoidance (CSMA/CA) period (SCP) that is segregated into many access windows (i.e., C-slot). The second part is a contention-free slotted TDMA period (STP) segregated into many T-slots [15]. Additionally, a hybrid CSMA/TDMA or queue-MAC has been developed to adjust to the varied traffic [16], where the contention-based CSMA was applied for a light load to address any delay due to scattered data transfer. Another MAC protocol based on TDMA was developed for gate-way multi-hop Wi-Fi-based long distance (WiLD) network in the attempt to enhance throughput and deal with the delay in performance [17]. This method was able to substantially reduce the possibility of collision for MAC protocol based on token through the use of synchronized inherent nodes approach. The suggested protocol enhanced the performance of the network by using the (available bandwidth to maximize the overlapping TDMA slots. The improvisation 
of MAC protocols was reflected by the average end-to-end delay in the packet for multiple hops, as well as saturated throughput. According to the best of our knowledge Dynamic Reservation (DR-TDMA) is one of the most efficient and comprehensive algorithm used in resource allocation for multimedia traffic in wireless network [18].

In particular, this approach includes the allocation algorithm that is based on an efficient rate of VBR video traffic which integrates algorithm to the control rate in order to ensure the flow of video with the related traffic thresholds. Hence, a 6bit piggybacking overhead was used to predicate the requirement for video connections in the previous protocols, In the last stage in each frame, DR-TDMA estimates the connection buffer status (with regard to number of packets, packets-guaranteed groups and best-effort) based on received data and parameters on connection, either immediately in control packet or piggybacked to data packet. Packets verified to video traffic threshold are referred as guaranteed packets with high QoS, whereas packets that fail to conform to the function on the basis of best-effort with the absence of QoS. In addition, the algorithm for slot allocation comprises two units, namely best-effort and guaranteed allocations.

The available slots were initially allocated by the scheduler to video connections awaiting guaranteed packets via time-toexpiry algorithm. The remaining slots were then allocated to best-packet video connection in virtue queue based on the algorithm for fair bandwidth allocation.

This study proposed the utilization of ODR-TDMA for video traffic. Hence, the primary aim of the suggested algorithm is to offer fair delay for the delayed video packets by reducing the variance in the delay between the transferred video packets. Since $\eta$ was modified, the corresponding value was met for each varied number of video user, which the ODRTDMA attempts to achieve through the creation of each frame. The control of the allocated resources or bandwidth is done in an adaptive manner for video traffic that correspond to the average bit rate as well as the ability to manage QoS with regard to packet loss, average delay and throughput.

This paper is organized as follows. Section II presents the system and traffic model description. Section III explains and discusses the proposed ODR-TDMA mechanism. The performance evaluation and simulation results have been reported in Section IV. Conclusion is presented in the final Section V.

\section{SYSTEM AND TRAFFIC MODEL DESCRIPTION}

The developed resource allocation algorithm can be utilized for slotted TDMA Wi-Fi, which functions by complying with the standards set in IEEE 802.11. The protocol was developed using Time Division Duplex (TDD). Fig. 1 shows the structure of frame used in the developed resource allocation algorithm.

The size for a frame of air interface was fixed at $2 \mathrm{~ms}$, and the frame was divided into equal-sized slots to moderate date scheduling with each slot having a 48-byte frame body.

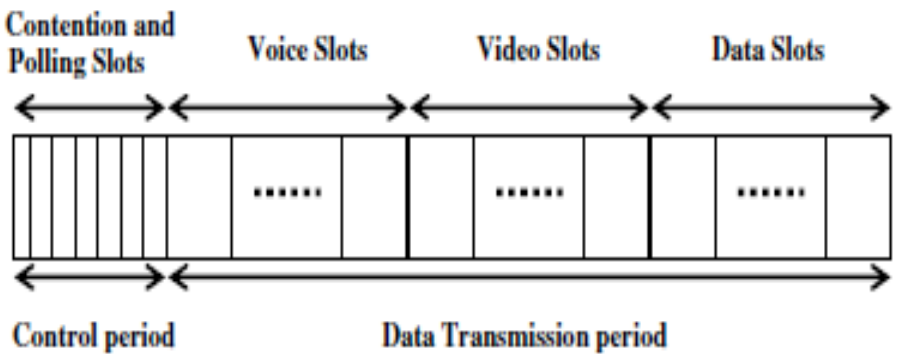

Fig. 1. Uplink Frame Structure.

\section{ODR-TDMA MECHANISM}

High usage of wireless channel and excellent QoS can be achieved through efficient resource allocation algorithm. In fact, the algorithm for transmission at uplink can also be easily used for the downlink scheduled by TDM. The upper and lower Delay_Thresholds were broadcast by base station to all video users in each frame created and their delay time in transferring packets is indicated by overhead piggybacking based on three categories: first, higher state for packet delay that is higher than upper Delay_Threshold; second, lower state for packet delay that is smaller than lower Delay_Threshold; and finally, In-betweens state for packet delay that is lower than upper Delay_Threshold but is higher than lower Delay_Threshold.

The latest updated status for packet delay is used by the allocation algorithm to manage slots for users at the end of each frame. This algorithm maximizes the amount of Inbetween delay state by converting lower and higher delay state into In-betweens state to obtain a fair delay.

The allocation algorithm depends on three steps. In the first step it assigns higher delay state, and increase number of allocated slots by 1 . The second step keep number of allocated slots without change. Finally, the third step assign users with delay lower than low delay threshold and reduce the number of allocated slots by 1 .

Since the lower delay state was the least affected by any decrease in the free available slots, it appeared in the final step. When the slot is absent, the user has to wait until an available slot is found. If the waiting required 3 frames, a slot is allocated by the base station in the present frame to deliver a packet and to update its delay state. Step 3 showed that users were distributed based on their wait state, where users with higher waiting time were served with fair allocation efficiently. Both the lower and upper Delay_Threshold were modified by the base station usingthe following equations [18]:

$D T H_{U}=D T H_{L}+D T H_{V A R} \quad$ (sec)

The Upper_Delay_Threshold $\left(D T H_{U}\right)$ is the summation of the Lower Delay threshold $\left(D T H_{L}\right)$, and the Variance Threshold $\left(D T H_{V A R}\right)$ is fixed at $4 \mathrm{msec}$.

$D T H_{L}=\frac{(R \operatorname{slot} * T f * E X)}{(N v * R v)} \quad(\mathrm{sec})$ 
Rslot is bit rate/slot at uplink channel bit rate for 24 slots/frame; $T f$ is the duration of the frame and is fixed at 2 $\mathrm{ms} ; E X$ is the number of additional slot for video traffic that exceeds the product of $\eta$ and $M ; N v$ is the number of video user; and $R v$ isaverage bit rate/video user.

$E X=E x+N o S-(\eta * M)($ slots $)$

$\eta$ is the weighting factor for the algorithm proposed in this study, $M$ is the number of slots that is equal to the total mean bit rate for all video users, and $N o S$ is the number of slots allocated for each user.

$M=(N v * R v) /$ Rslot (slots)

These equations suggest that the ODR-TDMA is able to modify the average number of slots allocated for video traffic near $M$ by regulating the values of lower and upper delay threshold. The upper and lower delay thresholds that are based on these equations were controlled more strictly by the base station when the EXCESS slot increased. Hence, lower delay state is used more often in comparison to higher delay state, thereby reducing the number of allocated slots. The values of Variance Threshold, which is the average of packet delay variance among the transferred packets, must be small, which is the reason for choosing $4 \mathrm{~ms}$ for 2 frames. The upper and lower Delay_Threshold values are equal to the Maximum Transfer Delay (MTD) video packets or $50 \mathrm{~ms}$ (maximum) and zero (minimum), respectively. The random value chosen by ODR-TDMA in the initial reading for $\eta$ was fixed as one the following values: $\{1.00,1.01,1.02,1.03,1.04\}$. It measured both packet delay and loss for each frame, and subsequently compared them with QoS, which was stored in the buffer upon adherence. With regard to the remaining frames, MPP was employed to scan the area and measure $N v$. The value of $N v$ was then compared with the values derived from prior processing to determine the suitable Lower and Upper Delay_Thresholds. With repetition, ODR-TDMA began to learn to identify the optimal value for $\eta$ which meet the requirements for QoS and adhere to the number of video users. Fig. 2 shows the flowchart for the developed ODR-TDMA for video traffic.

ODR-TDMA is able to obtain data from other wireless networks, therefore causing delay for users. To be precise, when MN failed to gain access to a WLAN network due to limited area of coverage, it can request for data, including the IP address of the Mesh Access Point (MAP) connected, to be sent to another network interface card. This MAP then sends an MN reply information message of neighbouring access networks (e.g. network id and channel numbers) that are within coverage. A list of available channels that operate in the available WLAN network was generated by the MN. If there was no WLAN network channel available within the coverage of the other network, the MN stayed in another network interface card, thereby allowing MPP access to the number of delayed users. All channels were scanned, either passively or actively, when the $\mathrm{MN}$ entered the interface card of the WLAN. If no MN was found, the interface card is switched off, but if an MN was found, the MPP will connect it with a new WLAN interface card to communicate data.

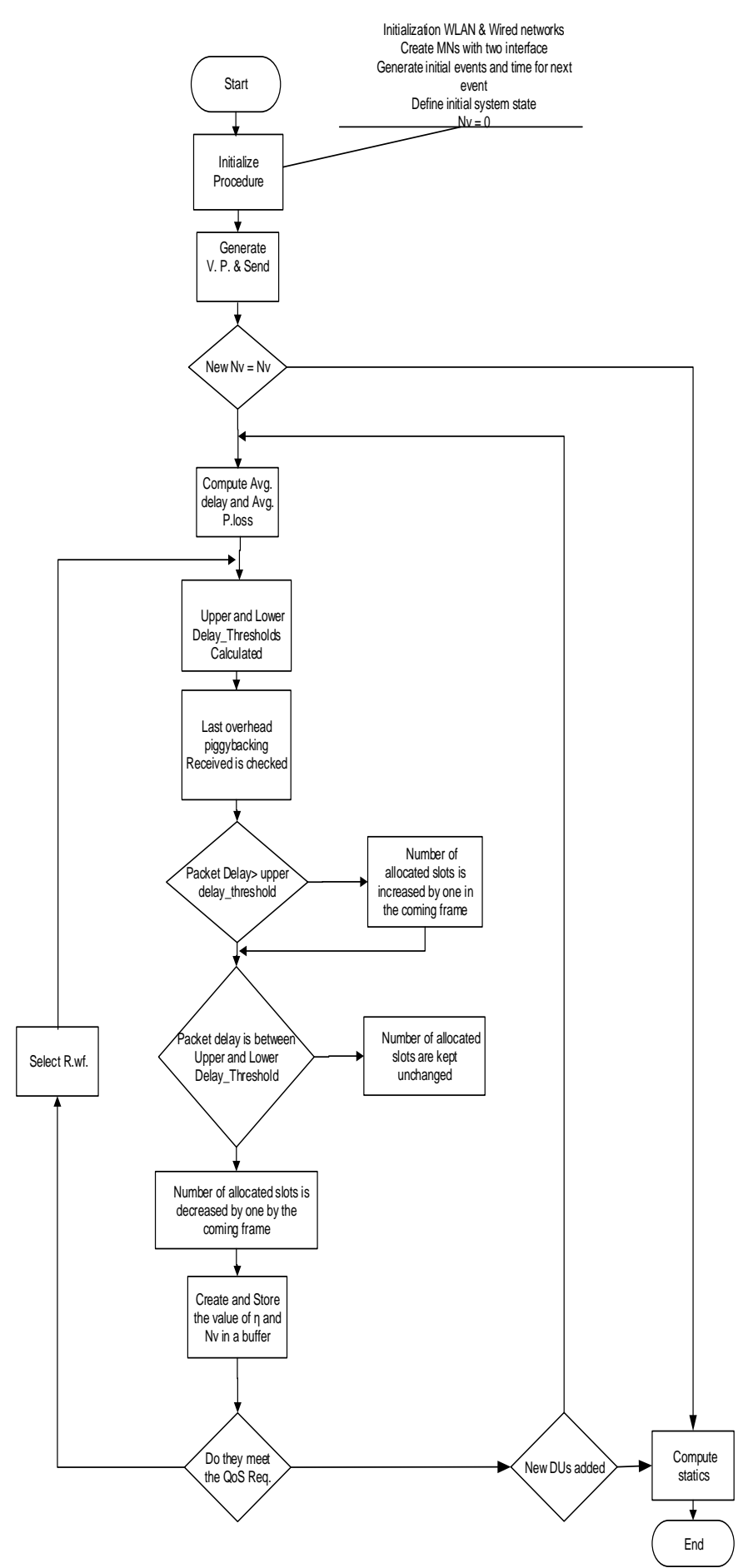

Fig. 2. ODR-TDMA Algorithm.

\section{Performance Evaluation And Simulation RESULTS}

Three primary metrics of performance were employed to evaluate the ODR-TDMA algorithm based on the alteration in MNs for the initial case and in $\eta$ for the second case. The outcomes from packet loss, packet delay, and throughput are presented and compared with the findings made in previous 
studies in two parts; the first part presents packet loss, average delay, and throughput when MNs were increased, whereas the second part shows a comparison of the outcomes for performance metrics between ODR-TDMA and Fair Dynamic Reservation- Time Division Multiple Access (FDR-TDMA) when MNs were increased.

\section{A. Effect of Increasing $N v$ when $\eta$ is within a Range of $\{1: 1.04\}$}

This section discusses the effect of ODR-TDMA when there was an increase in $\mathrm{MN}$ with the value for $\eta$ fixed at $\{1$ : $1.04\}$. The results for packet loss ratio, average delay, and throughput show the impact of ODR-TDMA in fulfilling the requirements of QoS. Fig. 3 shows that a small increase in $\eta$ could reduce packet loss that is proportional with the increase in both $\mathrm{Nv}$ and $\eta$. The expansion reflects the stability of $\eta$ as the value was gradually increased for Nv. It is interesting to note the small increase in $\eta$, as shown in Fig. 4 resulted in a substantial reduction in average packet delay. Efficient use of the allocated bandwidth by ODR-TDMA resulted in a substantial decrease in average packet delay and packet loss due to the change in value of Delay_Threshold. An increase in the MNs enhanced the dominance of $\eta$ that affects the increase in multiplexing gain, as well as a decrease in total variation rate for video traffic.

High values of $\eta$ have an impact a small group of users rather than a large group in attaining a similar QoS. Delay_Threshold is frequently influenced by the allocated slots and $\eta$. A reduction in allocated slots and an increase in $\eta$ tend to reduce the availability of free bandwidth, thereby generating higher delay state and reducing average packet delay due to the conversion to in-between state by ODR-TDMA.

Throughput is the average number of slot ratio for a successful transmission of data packet to the total amount of slots per frame. The similarities of the resulting throughput with $\eta$ are shown in Fig. 5. The second section presents a comparison of ODR-TDMA and FDR-TDMA. The video traffic channel parameters were fixed as recommended by [18].

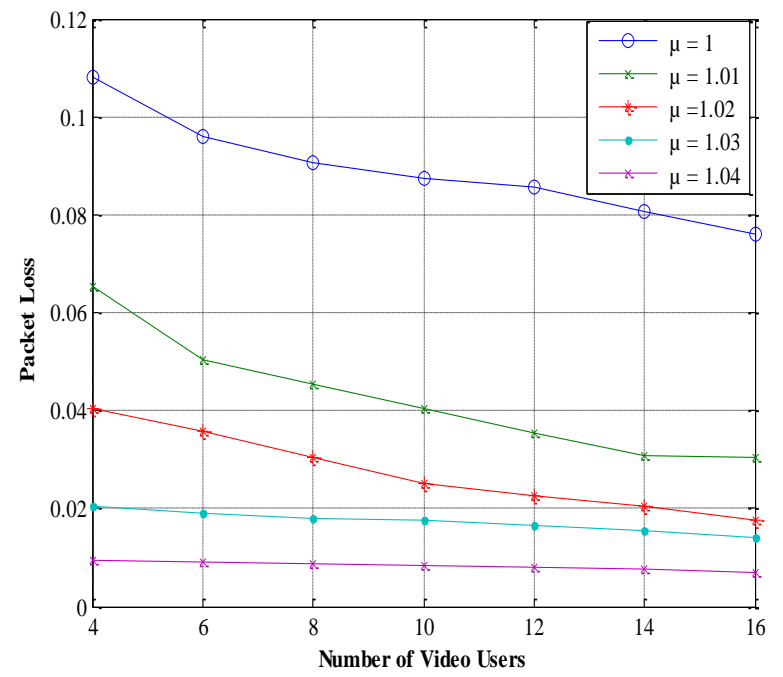

Fig. 3. Packet Loss Ratio as a Function of Nv.

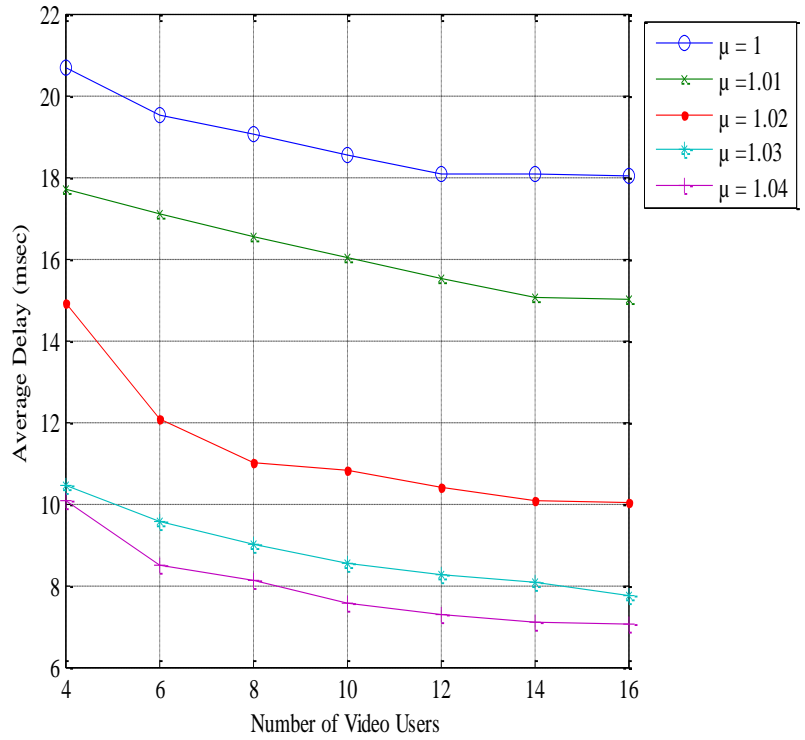

Fig. 4. Average Delay vs. No. of Video users.

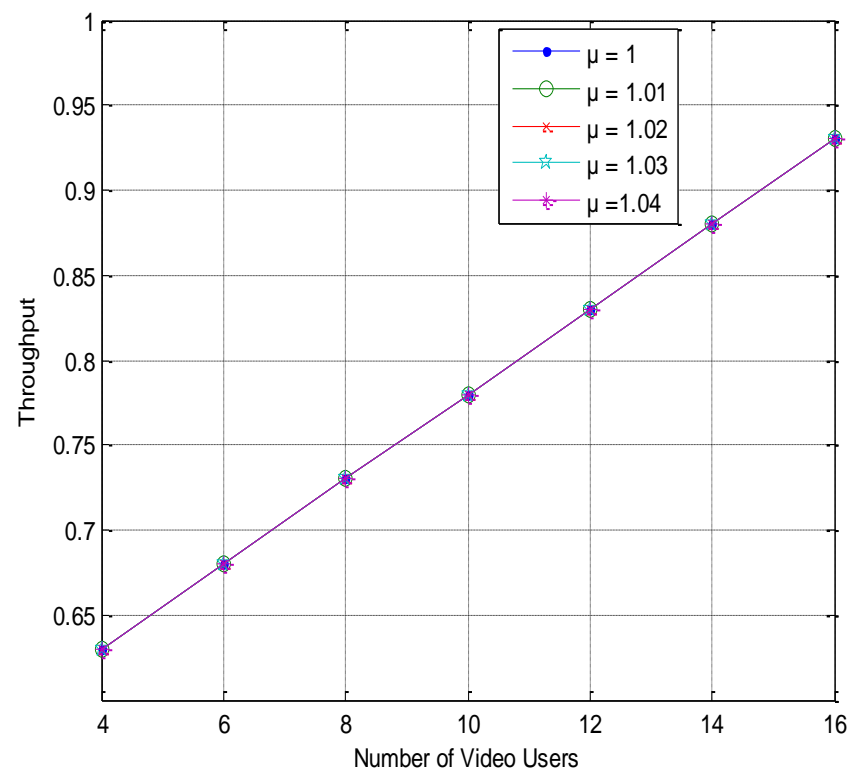

Fig. 5. Throughput vs. No. of Video users.

\section{B. Comparison of ODR-TDMA with Previous Protocols}

Channel parameters and users were set as recommended by [18] to compare video traffic simulations. The outcomes of the simulation of the packet loss ratio are shown in Fig. 6 to 9 . They show that the average values for throughput and packet delay for a video usder are $\eta=1.04$ and $250 \mathrm{Kbps}$, respectively. The first factor used to compare ODR-TDMA with other protocols was packet loss ratio. Fig. 6 shows a comparison with FDR-TDMA, which illustrates that ODRTDMA generated better outcomes by virtue of the reduced packet loss and the achieved consistency, which thereby gives exceptional QoS. The targeted 0.06 ODR-TDMA packet loss ratio supported 29 video users at a packet delay lower than 4 ms, where only 26 users were supported by FDR-TDMA. 


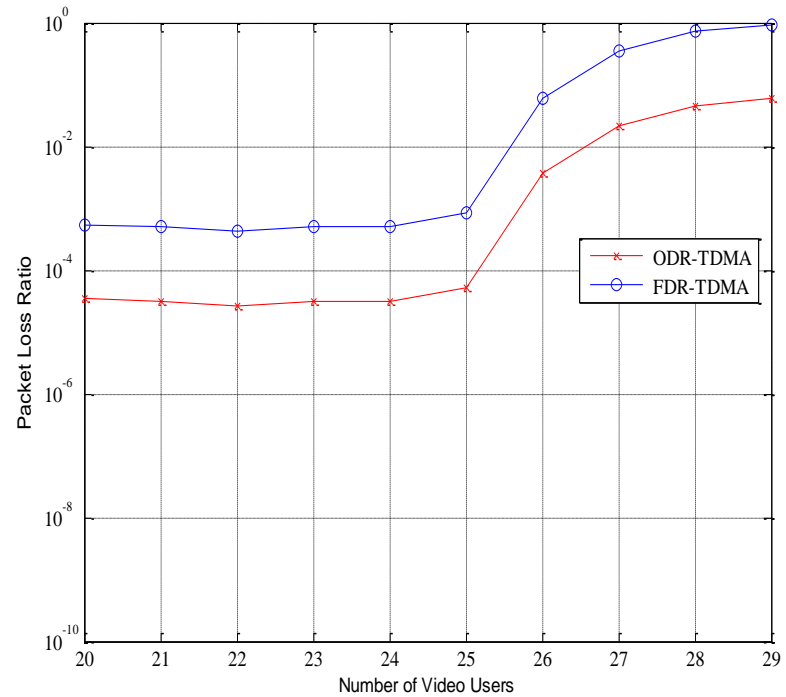

Fig. 6. Comparison of Packet Loss.

Fig. 7 illustrates that ODR-TDMA resulted in lower packet delay in comparison to FDR-TDMA, since it supported 20-23 users with a delay of less than $2 \mathrm{msec}$ whereas the delay in FDR-TDMA was $5 \mathrm{msec}$. By taking into account throughput and delay, the ODR-TDMA served 29 users with higher throughput, as can be seen in Fig. 8, in which the delay increased by only $3.25 \mathrm{msec}$ in comparison to FDR TDMA that served similar number of users with a delay of up to 19 msec.

Fig. 8 shows that ODR-TDMA exhibited slightly higher throughput when the system was overloaded. Both throughputs started at the same point and resulted in expansion, whereas $\mathrm{Nv}$ increased within a similar range up to $\mathrm{Nv}=26$, wherein ODRTDMA began to achieve higher throughput in comparison to FDR-TDMA.

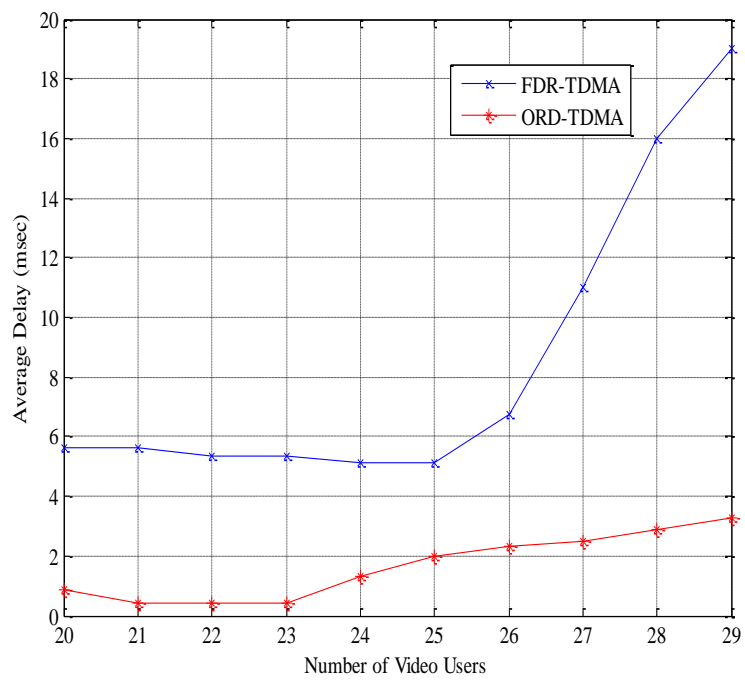

Fig. 7. Comparison of Average Delay.

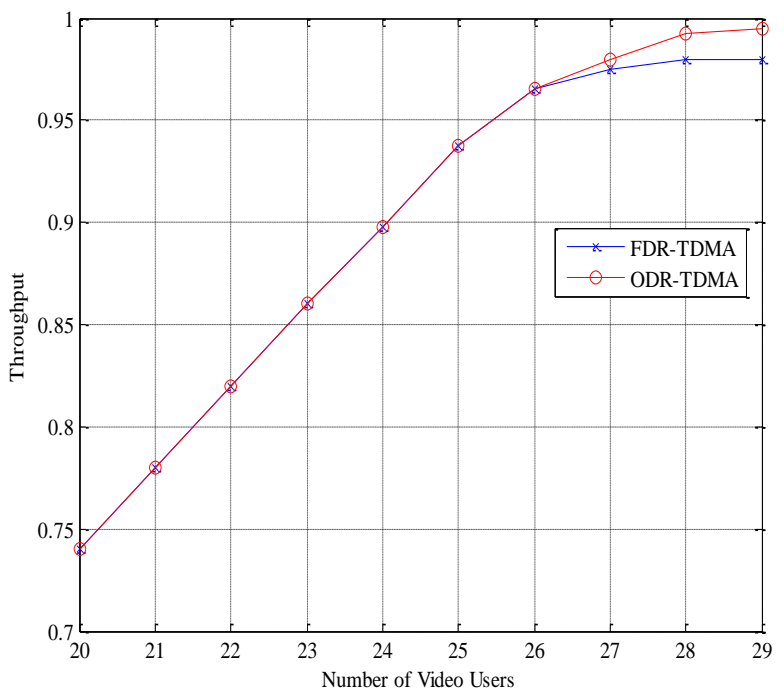

Fig. 8. Comparison on Throughput.

\section{CONCLUSION}

This study presents a unique resource allocation based on fair delay optimization for video traffic over WMNs system. As such, the recommended allocation algorithm was able to achieve the required QoS by reducing the variance in delay between the transmitted video packets in addition to flexibly controlling the allocated resource (bandwidth) for video traffic approximately the corresponding average bit rate that enhanced efficiency in its usage. The simulation results show an exception resource employment has achieved and offered an almost fair delay for the video packets. Moreover, it has the ability to manage the QoS for video traffic in terms of average delay and packets loss in order to generate exceptional QoS. In conclusion, the algorithm proposed in this study is successful in terms of improving packet loss, packet delay, and throughput in comparison to FDR-TDMA. For future work and as ODR-TDMA only concentrates and works with users who have higher delay than the higher delay threshold, a new algorithm could be developed to concentrate on users who have lower delay than the lower delay threshold and keep them in sleep mode during the waiting time so it can save the total consumption energy.

\section{REFERENCES}

[1] O. Kubbar, H.T. Mouftah, Multiple access control protocols for wireless ATM: problems definition and design objectives, IEEE Communication Magazine,1997, pp. 93-99.

[2] Y. Kwok, V.K.N. Lau, A quant itative comparison of multiple access control protocols for wireless ATM, IEEE Transaction on Vehicular Technology, 2001, pp. 796-815.

[3] I. Akyildz, J. McNair, L. Carrasco, R. Puigjaner, Medium access controls for multimedia traffic in wireless networks, IEEE Network Magazine, vol. 13, 1999, pp. 39-47.

[4] J. Wen, J. Lain, Y. lai, Performance simulation of wireless multimedia systems using NC-PRMA/DA and SNC-PRMA/DA protocols, IEEE Transactions on Systems, MAN, AND Cybernetics Part A: Systems and Humans, vol. 32, 2002, pp. 780-787. 
[5] Elnoubi, A.M. Alsayh, A packet reservation multiple access (PRMA)based algorithm for multimedia wireless system, IEEE Transaction on Vehicular Technology, vol. 53, 2004, pp. 215-222.

[6] J. Kuri, M. Gagnaire, ATM traffic management in an LMDS wireless access network, IEEE Communication Magazine, 2001, pp. 128-133.

[7] N. Passas, S. Paskalis, D. Vali, L. Merakos, Quality-of-service-oriented medium access control for wireless ATM networks, IEEE Communication Magazine, 1997, pp. 42-50.

[8] J.F. Frigon, H.C.B. Chan, V.C.M. leung, Dynamic reservation TDMA protocol for wireless ATM networks, IEEE Journal of Selected Areas in Communication, vol. 19, 2001, pp. 370-383.

[9] Jain, R.. Comparative analysis of contention based and TDMA based MAC protocols for wireless sensor networks. International Journal of Information Technology, 2018, pp.1-6.

[10] C.G. Kang, C.W. Ahn, K.H. Jang, W.S. Kang, Contention-free distributed dynamic reservation MAC protocol with deterministic scheduling (C-FD3 R MAC) for wireless ATM networks, IEEE Journal of Selected Area Communications, vol. 18, 2000, pp. 1623-1635.

[11] L. Musumeci, P. Giacomazzi, L. Fratta, Polling- and contention-based schemes for TDMA-TDD access to wireless ATM networks, IEEE Journal of Selected Area Communications, vol. 18, 2000, pp. 15971607.
[12] C.S. Chang, K.C. Chen, M.Y. You, J.-F. Chang, Guaranteed quality-ofservice wireless access to ATM networks, IEEE Journal of Selected Area Communications vol. 15, 1997, pp. 106-118.

[13] L. Fratta, P. Giacomazzi, L. Musumeci, PRAS: a Mac protocol for wireless ATM networks, Globecom'99, 1999, pp. 2743-2715.

[14] L. Lei, S. Cai, C. Luo, W. Cai, and J. Zhou, "A dynamic TDMA-based MAC protocol with QoS guarantees for fully connected ad hoc networks," Telecommunication Systems, vol. 60, 2015, pp. 43-53.

[15] Shahin, N., Ali, R. and Kim, Y.T., 2018. Hybrid Slotted-CSMA/CATDMA for Efficient Massive Registration of IoT Devices. IEEE Access.

[16] Zhuo, S., Wang, Z., Song, Y.Q., Wang, Z. and Almeida, L., 2016. A traffic adaptive multi-channel MAC protocol with dynamic slot allocation for WSNs. IEEE Transactions on Mobile Computing, vol 15, pp.1600-1613.

[17] Hussain, Md Iftekhar, Zaved Iqubal Ahmed, Nityananda Sarma, and D. K. Saikia. "An efficient TDMA MAC protocol for multi-hop WiFibased long distance networks." Wireless Personal Communications, vol. 86, 2016, pp. 1971-1994.

[18] Rizk, M.R.and et al., Fair Delay Optimization-based Resource Allocation Algorithm for Video Traffic over Wireless Multimedia System, Wireless personal communications, vol. 48, 2009, pp. 551-568. 